\title{
Sistema de monitoreo acelerográfico del Laboratorio de Ingeniería Sísmica
}

\section{Accelerographic Monitoring System of the Earthquake Engineering Laboratory}

\author{
Aarón Moya Fernández \\ Universidad de Costa Rica, Costa Rica \\ Docente e investigador del Laboratorio de Ingeniería Sísmica del \\ Instituto de Investigaciones en Ingeniería \\ cesar.moya@ucr.ac.cr
}

Recibido: 14 de octubre 2017

Aceptado: 23 de octubre 2017

\begin{abstract}
RESUMEN
El sistema automático de identificación y localización de sismos del Laboratorio de Ingeniería Sísmica de la Universidad de Costa Rica se empezó a desarrollar en el año 2010. Este procesa la información proveniente de más de 120 acelerógrafos digitales triaxiales conectados a la Internet. Ellos forman parte de la red de movimiento fuerte del laboratorio. Cuando ocurre un terremoto, el sistema calcula la localización y magnitud del sismo, así como los valores de aceleración y velocidad máximos, espectros de respuesta y diseño para cada una de las estaciones que se encuentren en línea en ese instante. El sistema funciona mediante módulos y el informe que se genera tarda alrededor de diez minutos. Sin embargo, cada vez que un módulo ha terminado de procesar los datos, la información de este se actualiza inmediatamente en el sitio web www.lis.ucr.ac.cr.
\end{abstract}

Palabras clave:

Acelerógrafo, sistema de procesamiento automático, ingeniería sísmica, terremoto, espectro.

\begin{abstract}
The Earthquake Engineering Laboratory at the University of Costa Rica has been operating an automatic earthquake processing system that started in 2010 . That system processes information gathered from over 120 digital triaxial accelerometers which are connected to the Internet. They are part of the laboratory's strong motion network. When an earthquake occurs, the system calculates the location and magnitude of the event as well as the peak ground acceleration and velocity, and the response and design spectra for each of the stations that are online at the moment. The system is written in modular programming, and the full report that is generated takes about ten minutes. However, whenever a module finishes processing the data, the information is immediately uploaded to the website at www.lis.ucr.ac.cr.
\end{abstract}


Keywords:

Strong motion accelerograph, automatic processing system, earthquake engineering, spectra, earthquake. 


\section{INTRODUCCIÓN}

El Laboratorio de Ingeniería Sísmica (LIS) de la Universidad de Costa Rica (UCR) cuenta en la actualidad con una red acelerográfica de más de 120 instrumentos distribuidos en todo el país. Estos son de tres marcas principales: Reftek, Nanometrics y Guralp. Todos son digitales, triaxiales y de 24 bits. Los acelerógrafos se encuentran ubicados en aulas u oficinas de centros educativos, comités locales de la Cruz Roja Costarricense, estaciones del Cuerpo de Bomberos, bibliotecas públicas y centros hospitalarios entre otros. Estos sitios son de un solo piso o máximo dos. En caso de edificaciones de dos pisos, los acelerógrafos son instalados en la planta baja.

Algunos de estos acelerógrafos se encuentran instalados en casetillas construidas específicamente para albergarlos, donde poseen electricidad y conexión a Internet. Estas casetillas miden $2 \mathrm{~m}$ x $2 \mathrm{~m}$ de lado y $3 \mathrm{~m}$ de alto con $1 \mathrm{~m}$ de profundidad. El equipo se encuentra sobre un pedestal de $0,5 \mathrm{~m}$ de alto en el centro de la casetilla.

Todos los acelerógrafos se hallan anclados al suelo mediante uno o más pernos (dependiendo de la marca: por ejemplo, en Reftek y Guralp se usa uno solo, en Nanometrics se usan tres). El perno se introduce dentro del concreto del primer piso de cada lugar y es ajustado. Todos los equipos son nivelados y conectados a su propia antena de GPS y a la Internet. Los datos son enviados en tiempo real a tres servidores ubicados en el Centro de Informática de la UCR donde se guardan con el respectivo formato de cada fabricante.

El desarrollo de la red acelerográfica del LIS y su sistema de procesamiento automático inició en el año 2010, cuando el laboratorio contaba con únicamente cuatro unidades digitales conectadas a Internet. En un principio, el sistema determinaba únicamente la aceleración y velocidad máximas, espectros de respuesta y comparaba los espectros de diseño de la norma vigente con los obtenidos durante el terremoto. El sistema se disparaba con la detección de un sismo fuerte en las cuatro estaciones que estuvieran en línea en ese momento y generaba un reporte que se subía de manera automática a la página web en www.lis.ucr.ac.cr.

En el mes de marzo del 2010, el número de equipos había crecido hasta alcanzar las 18 unidades. Para ese entonces, el sistema no solo calculaba los parámetros antes descritos, sino que también se inició el cálculo de la localización y magnitud de manera automática para todos los sismos.

En junio de ese mismo año, la red contaba con 43 unidades que abarcaban la mayoría del territorio nacional y se podían localizar sismos de magnitudes bajas (M 3 a 4). Sin embargo, en vista de que los parámetros de movimiento fuerte para sismos de baja magnitud tenían poco interés, se optó por limitar el procesamiento automático únicamente a los sismos que produjeran aceleraciones importantes. Es decir, en vez de procesar todo tipo de movimiento sísmico y calcular espectros para sismos débiles, se limitó el procesado para sismos de magnitudes moderadas a fuertes según fuera el valor de la aceleración del suelo.

Fue así como conforme la red iba incrementándose en número de equipos, el sistema de procesamiento automático también se fue volviendo cada vez más robusto y 
complejo con la incorporación de nuevos módulos. Al 2017, la red del LIS cuenta con 120 unidades digitales. Cada vez que ocurre un sismo lo suficientemente fuerte como para generar aceleraciones sensibles en más de 30 estaciones, el sistema se encarga de procesar la información acelerográfica, subir la información a la Internet y divulgarla por correo electrónico, mensajería de texto y redes sociales, sin intervención de un operario. Los diferentes módulos con que cuenta este sistema, permiten la estimación de los siguientes parámetros sismológicos e ingenieriles:

- Epicentro y magnitud con la distancia epicentral calculada respecto a 300 centros de población.

- Gráficos y tablas de valores máximos de los registros de aceleración para cada estación.

- Gráficos y tablas de velocidad máxima obtenida a partir de la integración de los registros de aceleración.

- Espectros de respuesta y comparación con los de diseño propuestos por la norma vigente.

- Mapa de fallas activas y amenaza sísmica con los valores de aceleración máximos registrados.

- Cálculo de la aceleración pico efectiva, intensidad de arias, velocidad acumulada máxima en cada estación acelerográfica (explicados más adelante).

- Resumen de los efectos del sismo en centros poblacionales sometidos a distintos grados de intensidad sísmica.

A este sistema de procesamiento automático se le ha dado el nombre de Sistema de Monitoreo Acelerográfico del Laboratorio de Ingeniería Sísmica (SMA-LIS).

\section{DETECCIÓN DE UN EVENTO FUERTE}

Los datos de aceleración provenientes de las tres marcas de equipos usadas por el LIS se guardan en el formato nativo que utiliza el fabricante de cada uno de ellos. Es decir, se utilizan las aplicaciones de fábrica para la transferencia y almacenamiento de la información. Así por ejemplo, en el caso de Reftek, el módulo que se encarga de la adquisición de los datos es el RTPD; en el caso de Nanometrics, se trata del ApolloServer; y en el de los equipos de marca Guralp, el SCREAM!. Cada grupo de equipos de una marca específica envía los datos a un servidor distinto en el Centro de Informática de la UCR. Los datos de los tres servidores son enviados a un cuarto que se encarga de homogeneizar toda la información en un único formato que es el Seismic Analysis Code o SAC (Goldstein, 2003).

Cada minuto, el SMA-LIS, que corre en el cuarto servidor, extrae la información acelerográfica del canal 2 (o componente NS) de los últimos $60 \mathrm{~s}$ de cada estación. Los datos en cuentas son convertidos a unidades de aceleración y guardados en formato SAC. Luego se aplica un filtro Butterworth de pasa bandas 0,1 a $10,0 \mathrm{~Hz}$ y se calcula el valor de aceleración pico (PGA por sus siglas en inglés). Este valor se compara luego con el PGA de los 2 minutos anteriores. 
El valor de PGA del último minuto se divide entre el valor del PGA del minuto anterior. Si el cociente es mayor o igual a un factor de 10, el nombre de la estación a que pertenece el registro se agrega a una lista de control. Este factor de 10 fue establecido mediante prueba y error y corresponde a un cambio de amplitud del PGA de la señal sísmica.

A esa lista de control se agregan todas las estaciones que cumplen con esa condición. Si el total de estaciones supera un umbral predefinido de 30, el SMA-LIS declara que se trata de un posible evento sísmico importante, basándose únicamente en el criterio del PGA. Este valor de 30 fue definido con el fin de que solo un movimiento lo suficientemente fuerte activara el sistema.

El sistema guarda el dato del tiempo de activación en un archivo de texto. A partir de ese momento, se crea una carpeta en el sitio web www.lis.ucr.ac.cr con la fecha y hora del tiempo de disparo donde se irán desplegando los resultados de todos los cálculos que se lleven a cabo. El primer gráfico que se prepara es el mapa de aceleraciones máximas registradas durante el último minuto. La figura 1 muestra el mapa de activación del sistema para un sismo ocurrido el 27 de julio del 2016. El evento tuvo una Mw 4,1 y se originó a la 04:50 a. m. El SMA-LIS creó el mapa al siguiente minuto, a las 04:51 a. $\mathrm{m}$. El número de estaciones que superó el umbral de 30, explicado anteriormente y que activó el sistema, fue de 50.

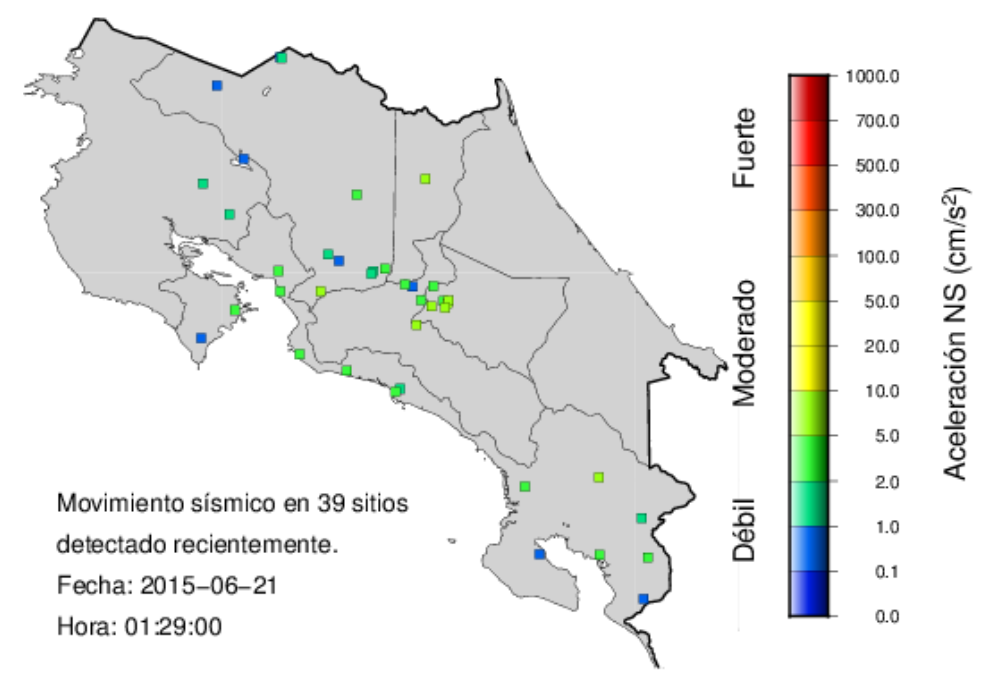

Figura 1. Ejemplo de mapa elaborado cuando el SMA-LIS se activa. Las estaciones que registraron el movimiento sísmico se muestran en distinto color según la escala mostrada a la derecha.

\section{PROCESAMIENTO DE UN SISMO}

Cuando se declara un evento sísmico, el sistema extrae los primeros 40 segundos previos al tiempo de disparo y los siguientes 3 minutos posteriores a este para todas las estaciones que se encuentran conectadas a la Internet, independientemente a que se hayan disparado o no. La información, en unidades de $\mathrm{cm} / \mathrm{s}^{2}$, se corrige por línea base y 
se filtra en el rango de 0,05-25,0 Hz. Nótese que este filtro es distinto al utilizado para el análisis de los datos de cada minuto, que tiene como fin el detectar un cambio en la amplitud del movimiento del suelo.

\subsection{Epicentro}

El SMA-LIS analiza todos los registros de las estaciones acelerográficas. Estaciones con registros que tengan un valor medio de aceleración inferior a $0,40 \mathrm{~cm} / \mathrm{s}^{2}$ en componente vertical, no son utilizadas para calcular el epicentro. La razón es que valores inferiores a $0,40 \mathrm{~cm} / \mathrm{s}^{2}$ poseen una amplitud muy baja y podrían afectar la selección del arribo de la onda P. Para el resto de estaciones, se aplica el siguiente procedimiento: Se toma el segundo actual y se calcula su valor absoluto. Luego se divide entre el valor absoluto del segundo anterior. Esto hace que se genere un pico grande cuando se detecta el inicio del sismo (figura 2). Esta evaluación segundo a segundo se aplica a todo el registro. En el momento en que ocurre el arribo de la onda $\mathrm{P}$, se generará un pico de amplitud superior al promedio tal como se ve en la figura 2. Luego de identificar el intervalo de tiempo donde se encuentra este valor máximo, se aplica la función APK de SAC (Goldstein, 2003) con el fin de determinar el tiempo exacto del arribo de la onda P. El cálculo del epicentro se realiza con el programa "elocate" (Herrmann, 2013) y el NonLinLoc (Lomax, 2000). El modelo de velocidades sísmicas utilizado es el obtenido por Quintero y Kissling (2001).

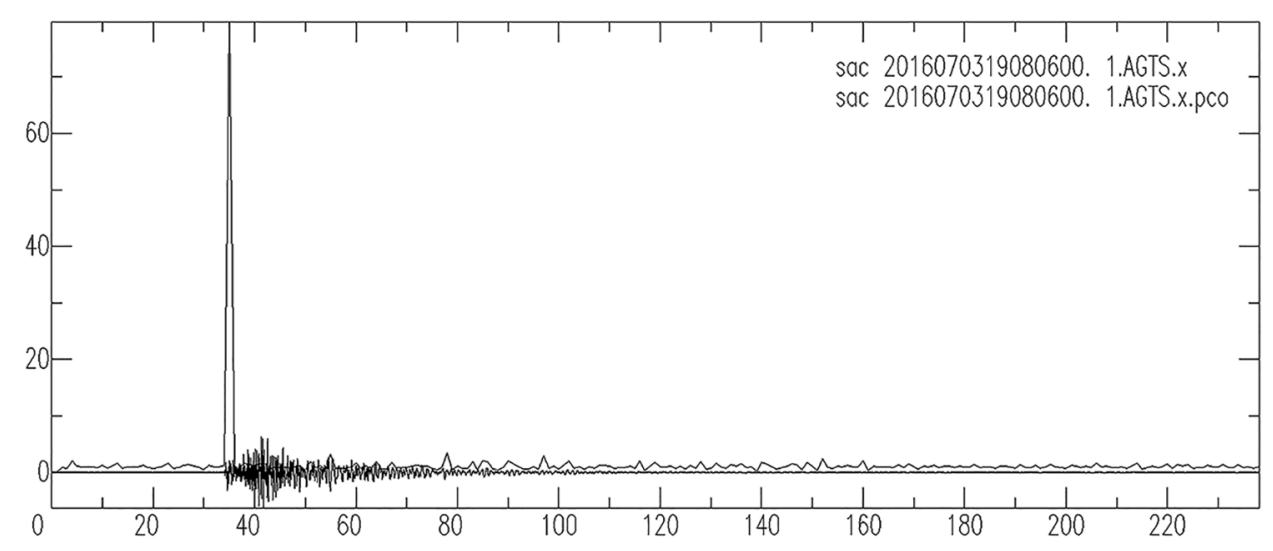

Figura 2. Ejemplo de la pre-selección del arribo de la onda P en un registro acelerográfico. La traza superior muestra el resultado del análisis que se hace segundo a segundo en la señal sísmica.

\subsection{Magnitud}

La magnitud se calcula utilizando los mismos registros que se usaron para determinar el epicentro del sismo. Se toma una ventana de $50 \mathrm{~s}$ previo al tiempo de arribo de la onda $\mathrm{P}$ en las componentes horizontales del registro. En igual forma, también se toma una ventana de 40 s posterior al tiempo de arribo de la onda P. A ambas ventanas se les aplica la transformada de Fourier para calcular el espectro de amplitud de la resultante horizontal, Os(f), según la siguiente ecuación: 


$$
O s(f)=\sqrt{N S(f)^{2}+E O(f)^{2}}
$$

donde

NS(f) es la componente norte-sur

EW(f) es la componente este-oeste

De manera análoga, se calcula la resultante horizontal para el ruido ambiente, $\operatorname{Or}(\mathrm{f})$. El espectro de amplitud de la aceleración se divide dos veces entre $2 \pi f$ para obtener el espectro de desplazamiento.

El espectro de la señal sísmica, Os(f), se compara con el del ruido ambiente, Or(f), en el intervalo 0,1 a $20 \mathrm{~Hz}$. Si la amplitud de la señal sísmica es 5 veces superior a la del ruido ambiente, el espectro de esa estación se utiliza para calcular el momento sísmico, Mo, usando la ecuación siguiente:

$$
M o=\frac{4 \pi \rho \Omega o \beta^{3} r}{R_{\theta \phi}}
$$

donde

$\rho \quad$ es la densidad del medio con un valor de $2700 \mathrm{~kg} / \mathrm{cm}^{3}$

$\Omega o \quad$ es el valor de la asíntota del nivel plano del espectro de desplazamiento

$\beta \quad$ es la velocidad de la onda cortante equivalente a $3200 \mathrm{~km} / \mathrm{s}$

$\mathrm{r} \quad$ es la corrección por distancia

$R_{\theta \phi} \quad$ es el patrón de radiación asumido $\sqrt{2 / 5}$ (Andrews, 1986).

El espectro de cada estación es corregido por el factor de superficie libre que tiene un valor de 2,0 y también por el factor de atenuación Q estimado por Moya (2009).

El SMA-LIS calcula la magnitud de manera independiente para cada una de las estaciones y toma el valor de la mediana en vez del promedio. La razón para utilizar la mediana en vez del promedio se debe a que el promedio se ve afectado por valores extremos. En caso de que una estación tuviera algún problema durante el procesado o que la señal sísmica fuera anómala (por ejemplo un problema eléctrico que causara amplitudes muy grandes o muy bajas), un valor muy alto podría dar una sobre-estimación de la magnitud. Esto no ocurre con el uso de la mediana.

Los datos del epicentro y la magnitud se muestran en la figura 3. Este es un sismo que no califica como un evento fuerte según la magnitud; sin embargo, ocurre en la zona central del país, donde la densidad de equipo acelerográfico es grande y por tanto, se 
registra fácilmente en más de 30 estaciones. Esto se debe precisamente a que el SMALIS utiliza los valores de aceleración como criterio de disparo.

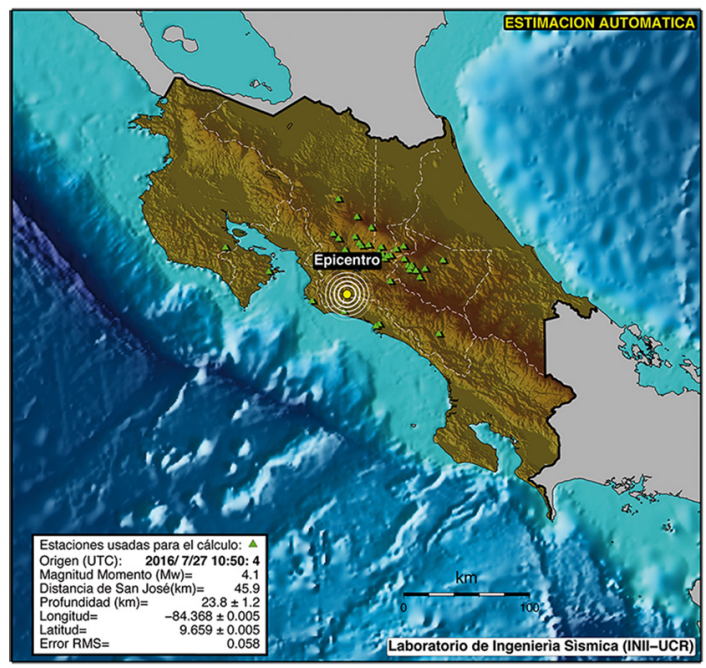

Figura 3. Ejemplo del mapa que se despliega en la página web del LIS (www.lis.ucr.ac.cr) cuando se activa el SMA-LIS y se calculan los parámetros de localización y magnitud de un sismo fuerte.

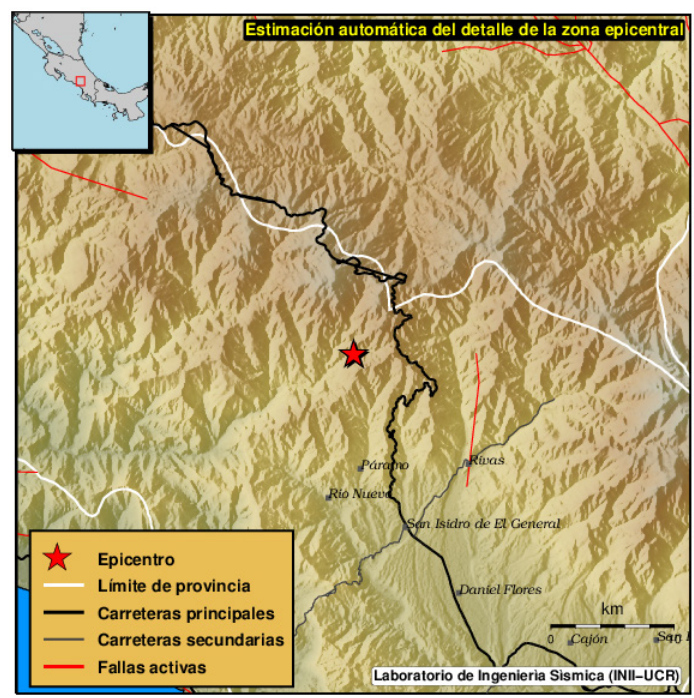

Ubicación del epicentro a los 10 lugares más cercanos.

Población

$9.2 \mathrm{~km}$ al N de Páramo de Pérez Zeledón

$11.7 \mathrm{~km}$ al N de Río Nuevo de Pérez Zeledón

$12.8 \mathrm{~km}$ al NO de Rivas de Pérez Zeledón

$14.5 \mathrm{~km}$ al NNO de San Isidro de El General Pérez Zeledón

$21.0 \mathrm{~km}$ al NNO de Daniel Flores de Pérez Zeledón

$21.8 \mathrm{~km}$ al NE de Tierras Morenas de Aguirre,Puntarenas

$29.1 \mathrm{~km}$ al NO de Cajón de Pérez Zeledón

$29.8 \mathrm{~km}$ al ESE de Santa María de Dota

$30.1 \mathrm{~km}$ al NE de Savegre de Aguirre

$32.2 \mathrm{~km}$ al SSE de Orosi de Paraiso

4511
3067
6593
46017
33591
52
8574
4621
3326
9092

Figura 4. Ejemplo de determinación de distancias epicentrales y mapa detalle para el sismo de la figura 3 . 
Además de esto, se calcula la distancia epicentral a los 10 centros de población más cercanos y se indica el número de habitantes que estas poseen, como lo muestra la figura 4. En el sitio web, el usuario puede escoger la ciudad de interés para conocer a que distancia se ubicó el epicentro desde ese centro poblacional. Se elabora también un mapa detalle del epicentro, en el cual se grafican las principales fallas activas (Denyer, Montero y Alvarado 2003).

\subsection{Aceleración y velocidad máxima}

Una vez determinados los parámetros de la fuente sísmica, los datos de todas las estaciones son utilizados para calcular los valores de aceleración y velocidad máxima (PGV por sus siglas en inglés). Los datos de velocidad se obtienen luego de integrar los registros de aceleración. En ambos casos, se tabulan los valores máximos de tres componentes por estación.

Los datos de aceleración máxima son también graficados en función de la distancia hipocentral, como lo muestra la figura 5. En este gráfico se superponen las curvas de atenuación calculadas para Costa Rica según el trabajo de Schmidt-Díaz (2014a).

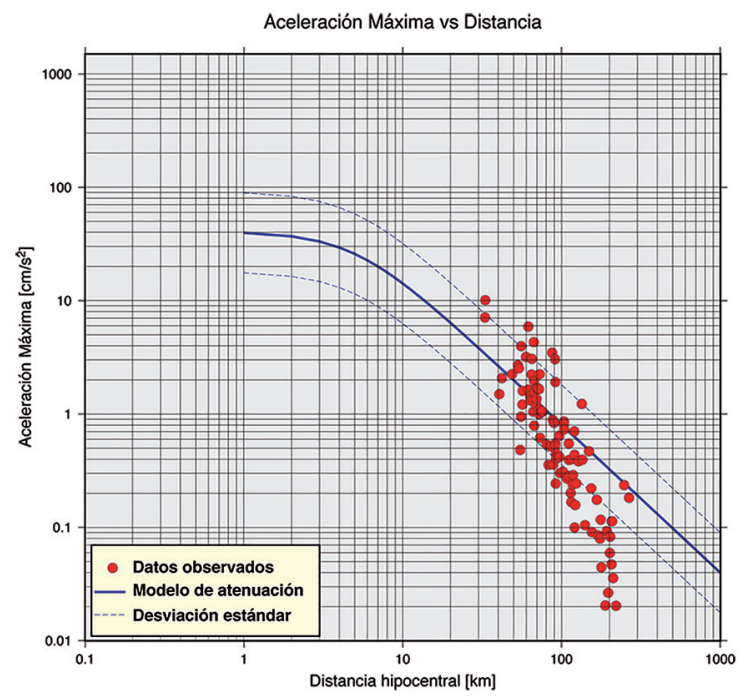

Figura 5. Gráfico de aceleración máxima vs distancia hipocentral para los valores de PGA de un sismo de Mw 4,2 ocurrido a $27 \mathrm{~km}$ de profundidad el 09 de setiembre del 2016.

En este apartado, el sistema también tabula el valor de PGA y PGV de la dirección transversal y radial. También se interpolan los valores de aceleración máxima y se muestran en un mapa. La figura 06 presenta el mapa de aceleración máxima interpolado para el sismo ocurrido el 01 de setiembre del 2017 de Mw 5.6 en Nosara.

El PGA suele ocurrir en un instante de tiempo muy corto, por esa razón, también se calcula el parámetro de la aceleración pico efectiva (APE) que se define como el promedio de las aceleraciones espectrales en un rango de periodos de 0,1 a 0,5 segundos, 
dividido por 2,5 (Applied Technology Council, 1984, 1995). La APE representa el movimiento del suelo en ese rango de periodos, que es donde se presentan los mayores valores de aceleración espectral. Este es el que propone el Código Sísmico de Costa Rica en su versión 2010 (Colegio Federado de Ingenieros y Arquitectos, 2010) como base para estimar las solicitaciones sísmicas en el país. La figura 7 muestra una gráfica de comparación entre el PGA y la APE que se crea de manera automática.

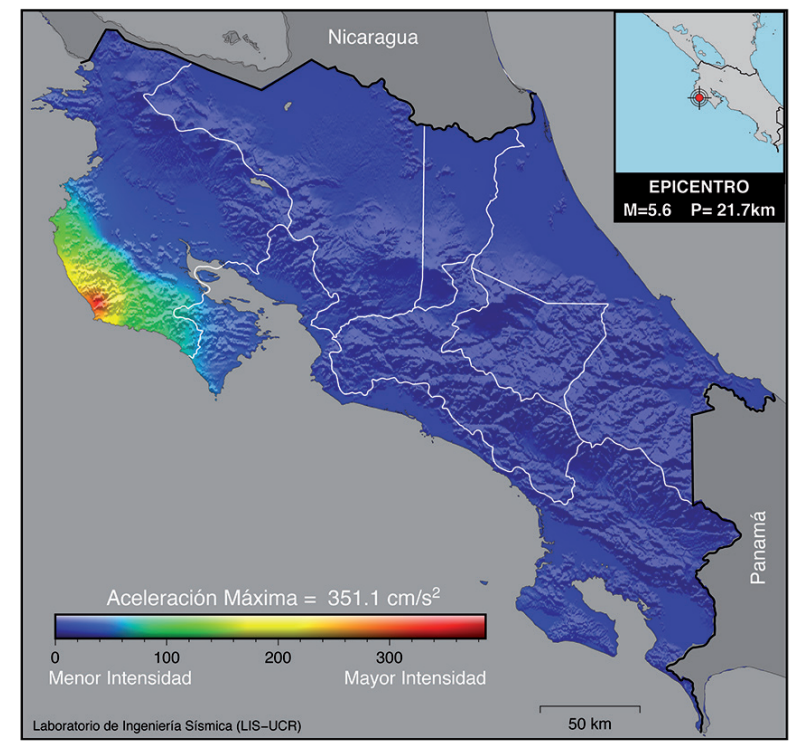

Figura 6. Mapa de aceleración máxima interpolada para el sismo ocurrido el 01 de setiembre del 2017.

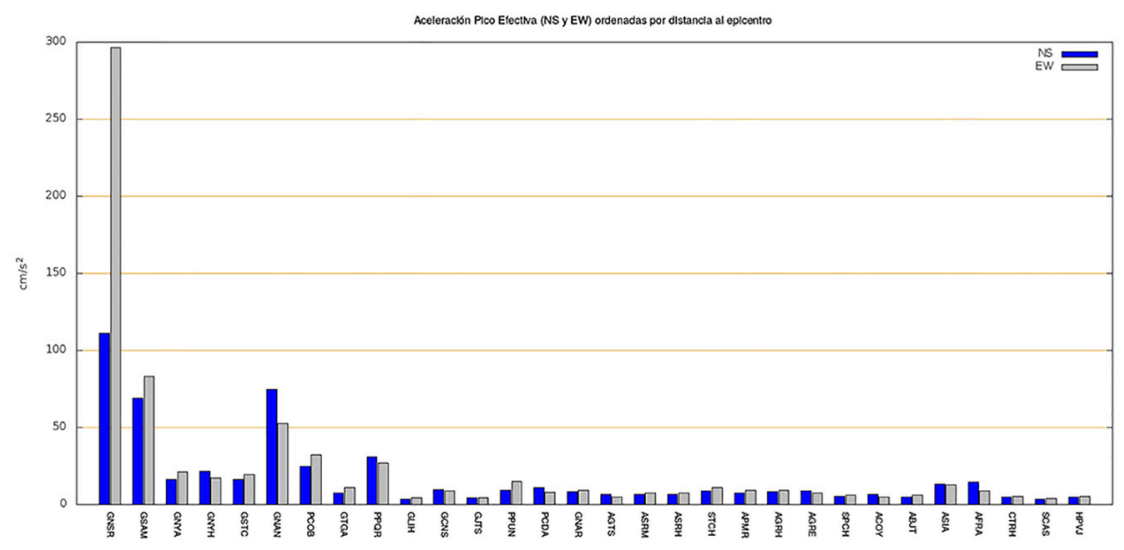

Figura 7. Valores de la APE en componente norte-sur (NS) y este-oeste (EW) para las 30 estaciones más cercanas al epicentro del sismo de la figura anterior. 
Además de la gráfica mostrada en la figura 7, el SMA-LIS compara también los valores de la APE con los del PGA y calcula el cociente APE/PGA para las 30 estaciones más cercanas al epicentro.

Otro parámetro de importancia es la Intensidad de Arias (Arias, 1970). La Intensidad de Arias (IA) es una medida instrumental que sirve para determinar los daños que un terremoto provoca en las estructuras y edificaciones. El SMA-LIS muestra el cálculo de la IA para la componente norte-sur (NS) y este-oeste (EW) de los registros de aceleración que tuvieron valores superiores a los $2,0 \mathrm{~cm} / \mathrm{s} 2$, así como el valor total de esta que corresponde a la sumatoria de componentes horizontales (Tselentis, 2011). La tabla 1 muestra los valores de la IA calculados para el mismo evento de la figura 7.

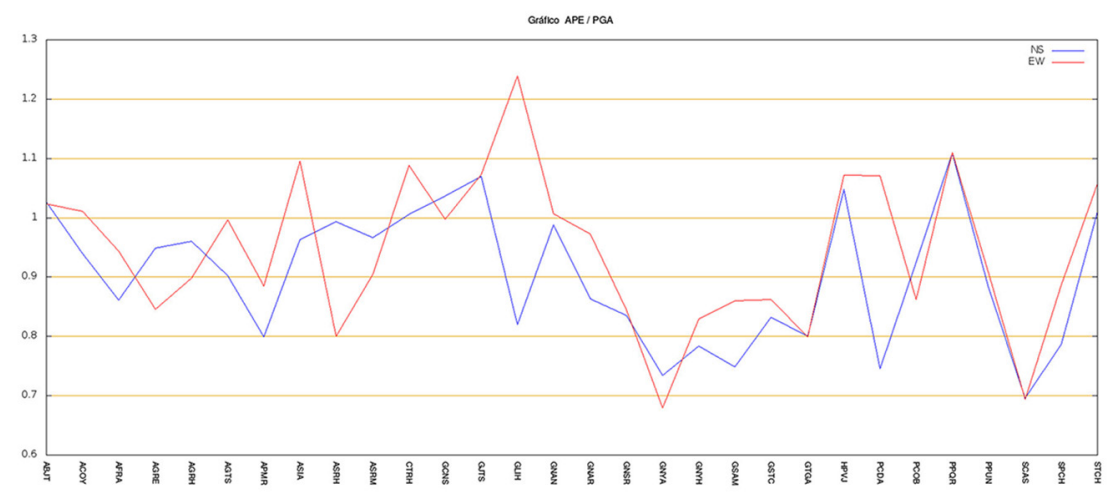

Figura 8. Gráfico del cociente de la APE/PGA para componente norte-sur (NS) y este-oeste (EW) para las 30 estaciones más cercanas al epicentro.

Tabla 1. Valores de IA para el sismo de la figura 7.

\begin{tabular}{cccc}
\hline Estación & IA (NS) & IA(EW) & IA (NS + EW) \\
\hline GNSR & 15.686840 & 58.222150 & 36.954495 \\
GNAN & 7.397609 & 4.356764 & 5.877187 \\
GSAM & 2.999480 & 3.531974 & 3.265727 \\
PPQR & 1.379290 & 1.065037 & 1.222163 \\
PCOB & 0.897924 & 1.165492 & 1.031708 \\
GSTC & 0.409064 & 0.662499 & 0.535782 \\
GNYH & 0.494441 & 0.395662 & 0.445052 \\
AGRH & 0.382294 & 0.468612 & 0.425453 \\
\hline ASIA & 0.327212 & 0.392611 & 0.359911 \\
\hline
\end{tabular}

Utilizando los velocigramas, el SMA-LIS calcula además el valor de velocidad acumulada absoluta (CAV por sus siglas en inglés) (Kramer, 1996) para componentes horizontales. 


\subsection{Espectros de respuesta}

Los espectros de respuesta son calculados para componentes horizontales de los registros acelerográficos. Estos se muestran en pseudoaceleración (PSA) en función del período. La figura 9 muestra un ejemplo del espectro de respuesta para la estación GNSR para el sismo del 01 de setiembre del 2017.

Además de estos espectros, a partir de enero del 2015, el sistema también crea una tabla resumen con los valores de PSA a los 0,3,1,0 y 3,0 s para todas las estaciones que tuvieron una aceleración superior a los $2,0 \mathrm{~cm} / \mathrm{s} 2$. Esta tabla muestra los valores máximos de cada componente. Es decir, aunque se calcula el PSA para esos tres períodos en las dos componentes horizontales, el sistema calcula el valor máximo de ambas y lo selecciona para mostrarlo en la tabla.
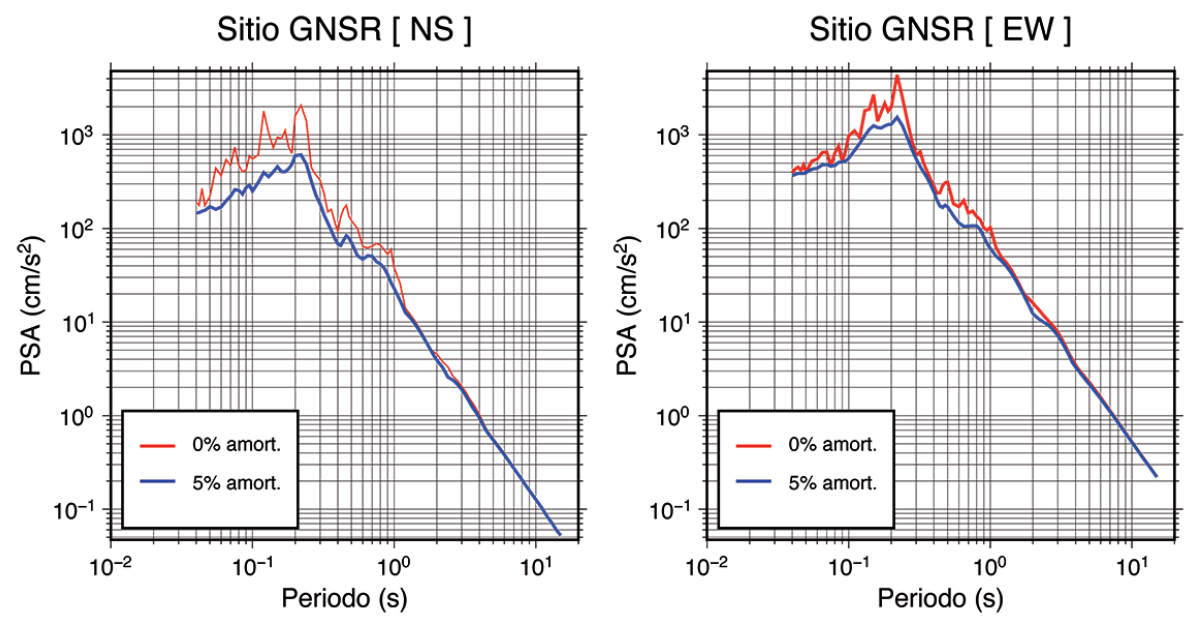

Figura 9. Ejemplo de un espectro de respuesta generado con el registro de componente norte-sur (NS) y este-oeste (EW) del evento del 01 de setiembre del 2017. La traza roja corresponde al espectro con $0 \%$ de amortiguamiento y la azul al del $5 \%$ de amortiguamiento.

\subsection{Espectros del Código Sísmico}

La zonificación sísmica es una guía de los valores mínimos que se deben considerar a la hora de diseñar estructuras. Además de las zonas, se debe también tomar en cuenta el tipo de suelo, sea este firme o blando. De acuerdo con el CSCR-2010, el país se divide en tres zonas sísmicas de importancia denominadas: Zona II, Zona III y Zona IV. Estas se muestran en el mapa de la figura 10. La Zona I es aquella en que los valores de aceleración del terreno son muy bajos por tratarse de rocas muy fuertes como intrusivos metamórficos o granitos. Este tipo de rocas es escaso en Costa Rica y por tanto dicha zona fue omitida del código.

Los valores de aceleración, según sea el tipo de suelo, se muestran en la misma figura en un cuadro inserto. Estos son S1 roca firme, S2 suelo firme, S3 suelo blando y S4 suelo muy blando. El SMA-LIS asigna el tipo de suelo a los sitios de las estaciones del LIS 
basándose en los resultados obtenidos por Schmidt-Díaz (2011, 2014b). El programa grafica la envolvente del CSCR-2010 y en el mismo gráfico superpone los espectros de los registros norte-sur y este-oeste, como lo muestra la figura 11.

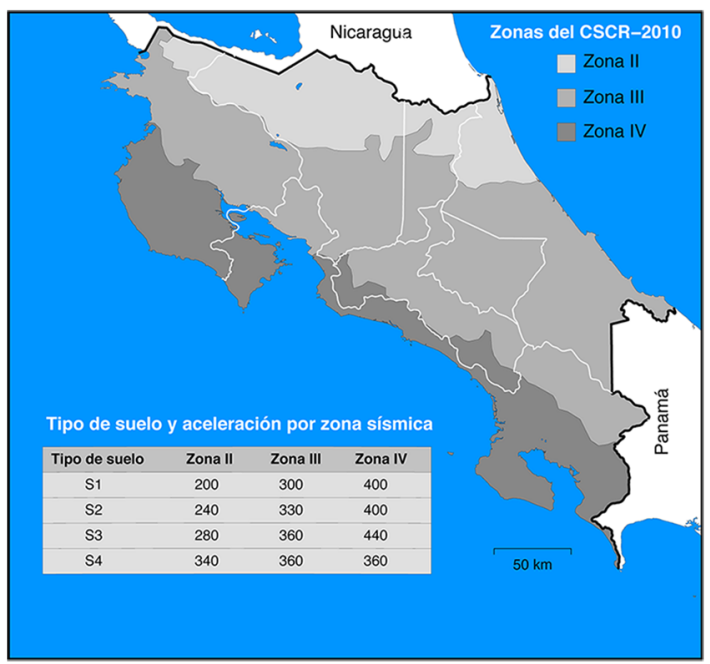

Figura 10. Zonificación sísmica de Costa Rica según el CSCR2010. El tipo de suelo corresponde a $\mathrm{S} 1=$ roca, $\mathrm{S} 2=$ firme, S3=blando y S4=muy blando. Los valores de aceleración están dados en $\mathrm{cm} / \mathrm{s} 2$.

\section{Sitio LTAL}

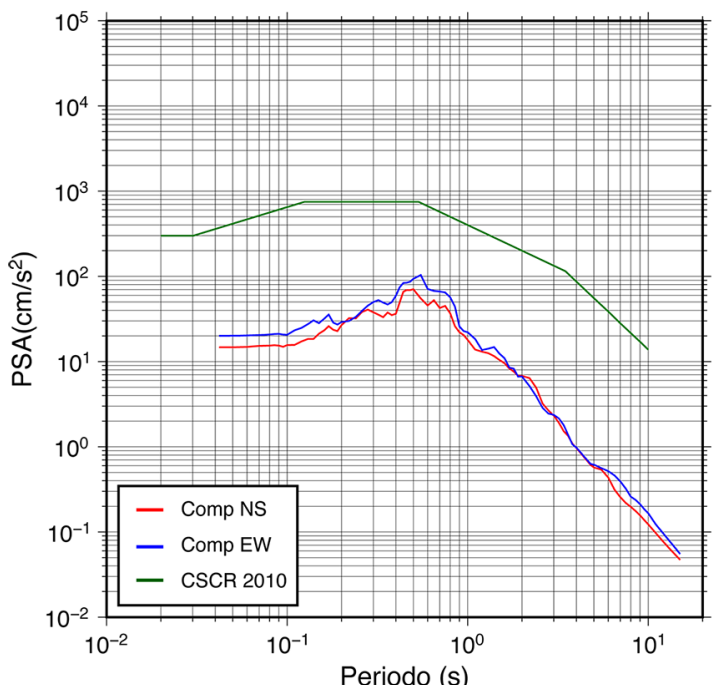

Figura 11. Gráfico del espectro propuesto del CSCR-2010 (traza verde) y espectros de componentes horizontales norte-sur (NS) y este-oeste (EW) en la estación LTAL para un sismo ocurrido el 02 de abril del 2017 con epicentro en Panamá y Mw 5,6. 


\section{EFECTOS SOBRE LA POBLACIÓN}

Antes del año 2017, el SMA-LIS asignaba valores de intensidad sísmica en la escala modificada de Mercalli. Se usaban las relaciones empíricas obtenidas por Wald, Quitoriano, Heaton y Kanamori (1999). Sin embargo, a partir de ese año, se optó por calcular la intensidad propuesta por la Agencia Meteorológica de Japón (JMA) (1996), llamada Shindo. Esta describe el grado de agitación en un punto de la superficie terrestre. Se calcula a partir de las tres componentes de un registro de aceleración y de la duración de la sacudida. La escala varía de 0 a 7 , donde 0 es un movimiento imperceptible y 7 corresponde a un terremoto destructivo. La figura 12 muestra el mapa de intensidades sísmicas obtenido para el sismo ocurrido el 02 de abril del 2017 en Panamá (Mw 5.6). Este evento produjo un valor de intensidad en la escala Shindo 4.

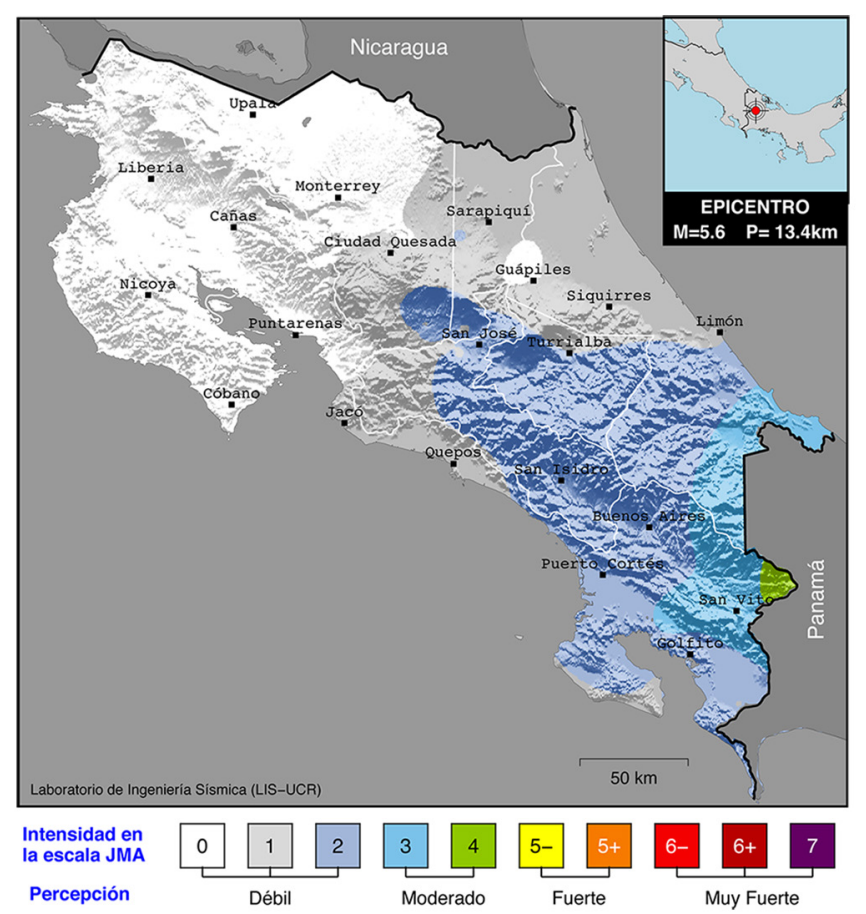

Figura 12. Mapa de intensidades sísmicas en la escala JMA para un sismo con epicentro en Panamá. El SMA-LIS calcula la intensidad Shindo usando las 3 componentes de aceleración de cada registro y la duración de los mismos. En la parte de abajo se muestran los valores de la escala que varían de 0 a 7. El valor máximo para ese sismo fue de Shindo 4.

A partir del mapa de intensidades, se utilizan las funciones del programa Generic Mapping Tools (GMT) (Wessel, Smith, Scharroo, Luis y Wobbe, 2013) para identificar la ubicación de los centros de población en una zona de intensidad sísmica específica. El SMA-LIS hace un recuento de estos y genera un mapa a color y con un recuadro que muestra el número de centros en cada zona isosísmica. Siguiendo con el mismo ejemplo del mapa de la figura 12, el mapa de los centros de población sería el de la figura 13. 
Las líneas blancas en el mapa de la figura 13 son las divisiones por cantones. Toda esta información se brinda en formato HTML así como en PDF bajo el apartado "Efectos sobre la Población" en el informe que se encuentra en línea. También en línea se pueden consultar de manera dinámica las intensidades sísmicas asignadas a cada distrito.

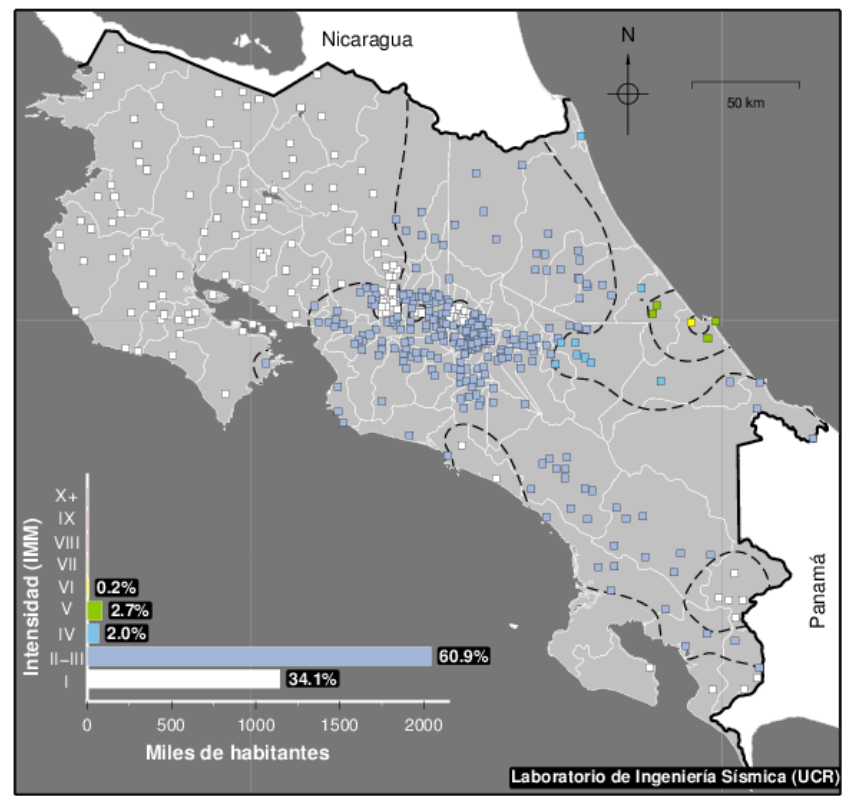

Figura 13. El sistema automático calcula el número de habitantes que hay en cada centro de población (indicados por cuadros en el mapa). Cada centro se colorea según haya sido el valor de la intensidad sísmica experimentado. Además, tomando el número de habitantes que hay en cada uno, se muestra el porcentaje de habitantes que viven en las distintas zonas isosísmicas para este evento en particular.

\section{DISCUSIÓN}

Uno de los objetivos del SMA-LIS cuando fue desarrollado fue contar con un instrumento de procesamiento automático para la gran cantidad de datos acelerográficos que se transmitían al LIS. Tal como se mencionó en la introducción, el desarrollo del software fue gradual y respondía a necesidades específicas del laboratorio que se han ido incorporando en la manera de módulos.

Estos módulos ejecutan programas de procesamiento de señales estándar, como las funciones para la selección de los tiempos de arribo y cálculos de espectros del software SAC (Goldstein, Dodge, Firpo y Minner, 2003). Además de procesar la información, el SMA-LIS guarda copia de los registros originales para que la información extraída de manera automática pueda ser utilizada por los investigadores en el momento de revisar los cálculos o llevar a cabo nuevas investigaciones. Los resultados obtenidos por el SMA-LIS, lejos de ser tomados como valores conclusivos, deben ser más bien vistos 
como cálculos preliminares que podrán ser validados por investigaciones futuras pero que brindan información de primera mano luego de la emergencia.

Los valores de la localización de la fuente sísmica y el cálculo de la magnitud deben ser vistos con un valor agregado al del procesamiento acelerográfico del SMA-LIS. Estos son posibles debido a que los equipos están conectados las $24 \mathrm{H}$ a la Internet y cada uno posee un sistema de GPS, a diferencia del pasado, en que los acelerógrafos se disparaban únicamente cuando había una sacudida fuerte que sobrepasara un umbral predefinido.

La figura 14 muestra los epicentros de todos los sismos detectados de manera automática por el sistema desde el año 2010.

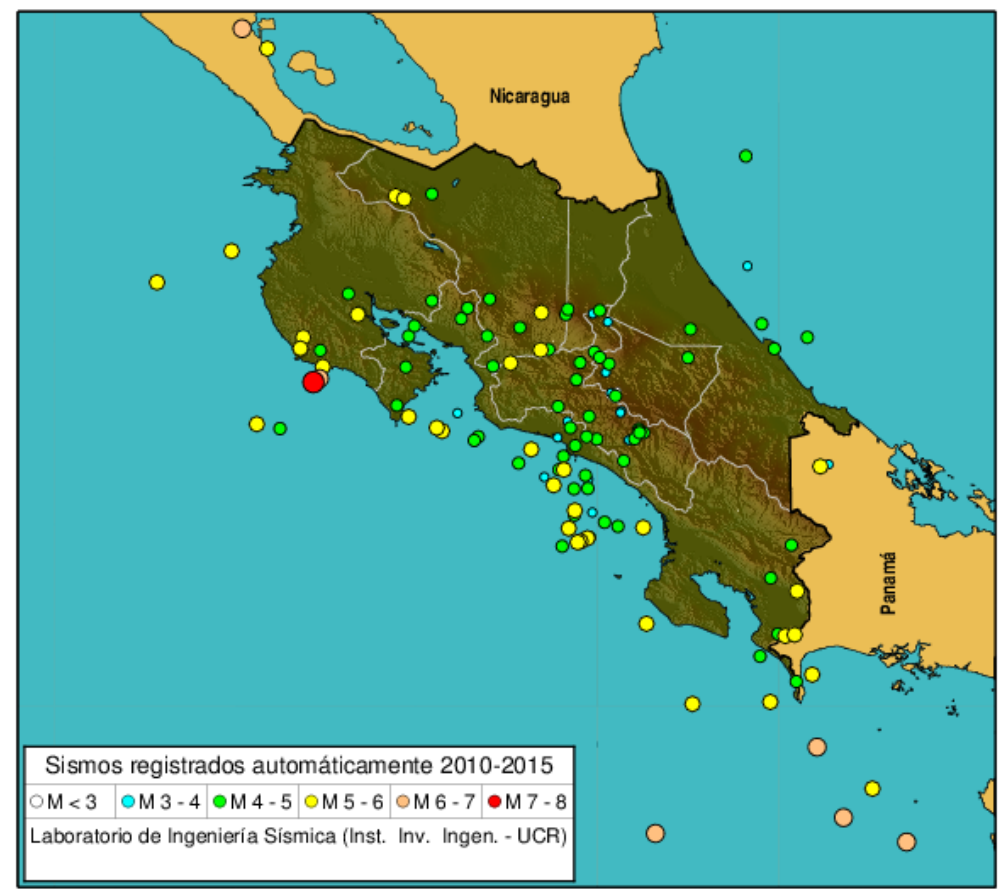

Figura 14. Epicentro de los sismos que el SMA-LIS ha procesado de manera automática.

Existen otros cálculos menores que también se realizan y que no se han detallado en este artículo como el de superponer los datos de la aceleración máxima sobre los mapas de amenaza sísmica y el mapa de fallas activas del Cuaternario (Denyer et al., 2003) o el del cálculo de los efectos de sitio de todas las estaciones aplicando la técnica $\mathrm{H} / \mathrm{V}$ y comparando el valor del cociente del sismo actual con el del promedio.

El sistema tiene, por supuesto, sus debilidades. Por ejemplo, este ubicó de manera correcta el epicentro del sismo del 05 de setiembre del 2012 frente a las costas de Sámara de Nicoya, provincia de Guanacaste. Sin embargo, subestimó la magnitud del sismo que era de Mw 7.6 y el sistema lo calculó en Mw 7,0.

El sistema es también vulnerable a los sismos fuertes ocurridos a distancias considerables. El ejemplo más reciente fue el del sismo del 14 de octubre del 2014 con 
epicentro frente a las costas de Nicaragua y El Salvador. La magnitud del sismo fue de Mw 7,3, pero el movimiento sísmico fue percibido en la parte norte de Costa Rica. Esto activó el sistema que, debido a la lejanía del epicentro, lo ubicó frente a las costas de Guanacaste, donde la cobertura de estaciones era escasa. Aun así, el epicentro y la magnitud del sismo tienen poca relevancia en relación a la información acelerográficas que se procesa (como lo son los valores de PGA y el cálculo de los espectros), por lo que estos parámetros pueden luego ser ajustados con la información que brindan instituciones sismológicas nacionales o internacionales.

También se han presentado errores en la localización de sismos que ocurren a lo largo de la Zona de Fractura de Panamá, al sur del país. Nuevamente el problema es la baja cobertura de estaciones fuera de las fronteras costarricenses.

\section{CONCLUSIONES}

El sistema que opera en el LIS para el procesamiento de los datos acelerográficos, como todo programa de computación, se encuentra en constante evolución. Los módulos que se explicaron en este artículo son revisados cada vez que ocurre un sismo fuerte con el fin de mejorar el procesamiento de la información. Los cálculos de la magnitud, por ejemplo, son comparados con los de otras instituciones luego de un movimiento importante. Todos los registros de aceleración son inspeccionados de manera visual luego de un terremoto. Cuando se detecta un error en alguno de ellos, este es removido del informe automático y el documento es actualizado.

El sistema del LIS ha estado en funcionamiento desde el año 2010 y ha registrado de manera satisfactoria un total de 130 eventos moderados a fuertes. El más grande de ellos fue el de Mw 7,6 en el 2012. Los sismos de magnitudes relativamente bajas, pero cercanos a estaciones acelerográficas, han producido aceleraciones altas que han activado el sistema. En el año 2014, tres sismos de magnitudes 4,3, 4,4 y 4,1 ocurrieron cerca de la capital, donde se ubican la mayor cantidad de acelerógrafos. Uno de ellos produjo aceleraciones de $245 \mathrm{~cm} / \mathrm{s} 2$ que activó el sistema automático.

El SMA-LIS ha mostrado ser una herramienta de gran utilidad, tanto para los encargados de la toma de decisiones en materia de atención de emergencias y como para la comunidad ingenieril. Por ejemplo, luego de ocurrido el terremoto de $\mathrm{Mw}$ 7,6 del 2012 con epicentro en el Pacífico, se generó el mapa de intensidades sísmicas que rápidamente permitió identificar una región al oeste de la capital donde las aceleraciones habían sido particularmente altas. Esto fue debido a la amplificación sísmica producto de los suelos relativamente blandos de origen lacustre que predominan en la zona (Rojas, 2013).

Debido a la arquitectura modular en que se ha implementado la programación del SMA-LIS, este permite que se puedan añadir fácilmente otros cálculos. Por ejemplo, se trabaja en la incorporación de módulos de probabilidad de daño para estructuras de importancia que se encuentren cerca de los sitios de registro como las instituciones educativas y de atención de emergencias que son las que, en su mayoría, resguardan los equipos acelerográficos. 
Los datos de aceleración de cada estación se guardan en formato binario junto con el informe que se muestra en línea. Sin embargo, se pretende incorporar otro módulo que los convierta en formato de texto simple (ASCII). Esto con el fin de que los registros puedan ser accesados de manera inmediata por estudiantes e investigadores que usan programas de procesamiento distintos a los de los fabricantes de los acelerógrafos.

\section{AGRADECIMIENTOS}

Este trabajo ha sido posible gracias al financiamiento que recibe el Laboratorio de Ingeniería Sísmica a través de la Ley Nacional de Emergencias y los proyectos de investigación inscritos en la Vicerrectoría de Investigación de la Universidad de Costa Rica. Se agradece a los técnicos Esteban López, Esteban Cordero y Carlos Segura por el trabajo de instalación y mantenimiento de la red del LIS.

\section{REFERENCIAS}

Andrews, D.J.(1986). Objective determination of source parameters and similarity of earthquakes of different size. Geophysical Monographs, 37(6), 259-267.

Applied Technology Council (1984). Tentative Provisions for the Development of Seismic Regulations for Buildings, ATC-3-06. California: National Bureau of Standards.

Applied Technology Council (1995). A Critical Review of Approaches to Earthquake Resistant Design, ATC-34. California: National Bureau of Standards.

Arias, A. (1970). A Measure of Earthquake Intensity. En R.J. Hansen (ed.) Seismic Design for Nuclear Power Plants (pp. 438-483). Massachusetts: MIT Press.

Colegio Federado de Ingenieros y de Arquitectos (2010). Código Sísmico de Costa Rica. Costa Rica: Editorial Tecnológica de Costa Rica,.

Denyer, P., Montero, W., y Alvarado, G. (2003). Atlas Tectónico de Costa Rica. Costa Rica: Editorial Universidad de Costa Rica.

Goldstein, P., Dodge, D., Firpo, M., y Minner, L. (2003). SAC2000: signal processing and analysis tools for seismologists and engineers. En W. H. K. Lee, H. Kanamori, P. C. Jennings C. Kisslinger (eds.) The IASPEI International Handbook of Earthquake and Engineering Seismology. London: Academic Press.

Herrmann, R. B. (2013) Computer programs in seismology: An evolving tool for instruction and research. Seism. Res. Lettr. 84, 1081-1088. doi:10.1785/0220110096

Kramer S. L. (1996). Geotechnical Earthquake Engineering. New Jersey: Prentice-Hall.

Moya, A. (2009). Inversión de efectos de sitio y factor Q utilizando cocientes espectrales. Estudios Geológicos, 65(1), 67-77.

Quintero, R. y Kissling, E. (2001). An improved P-wave velocity reference model for Costa Rica. Geofísica Internacional, 40(1), 3-19.

Rojas, V. (2013). Relación entre los procesos Volcano-Sedimentarios y el Neotectonismo de la Cuenca Lacustrina de Palmares y San Ramón, Costa Rica. [Tesis de Licenciatura]. Escuela Centroamericana de Geología, Universidad de Costa Rica, Costa Rica. 
Schmidt-Díaz, V. (2011). Clasificación de suelos basada en el cálculo de razones espectrales en sitios donde se ubican estaciones acelerográficas de América Central. Casos de El Salvador, Nicaragua y Costa Rica. Rev. Geol. Amér. Central, 44, 9-26.

Schmidt-Díaz, V. (2014a). Ecuaciones predictivas del movimiento del suelo para América Central, con datos de 1972 a 2010. Rev. Geol. Amér. Central, 50, 7-37.

Schmidt-Díaz, V. (2014b). Clasificación de suelos de 15 estaciones acelerográficas, mediante el uso de métodos basados en vibraciones ambientales y del parámetro VS30. Rev. Geol. Amér. Central, 51, 33-67.

Tselentis, G-A. (2011). Assessement of Arias intensity of historical earthquakes using modified Mercalli intensities and artificial neural networks. Nat. Hazards Earth Syst. Sci., 11, 3097-3105.

Wald, D.J., Quitoriano, V., Heaton, T.H., y Kanamori, H. (1999). Relationship between Peak Ground Acceleration, Peak Ground Velocity, and Modified Mercalli Intensity in California. Earthquake Spectra, 15(3), 557-564.

Wessel, P., Smith, W. H. F., Scharroo, R., Luis, J. y Wobbe, F. (2013). Generic Mapping Tools: Improved version released. EOS Trans. AGU, 94, 409-410. 